\title{
An Optimizing Technique based on Genetic Algorithm for Power Management in Heterogeneous Multi-Tier Web Clusters
}

\author{
Pankaj Goyal \\ Student \\ University Institute of Engineering and Technology \\ Panjab University, Chandigarh
}

\author{
Nirmal Kaur \\ Assistant Professor \\ University Institute of Engineering and Technology \\ Panjab University, Chandigarh
}

\begin{abstract}
The most serious drawback nowadays is the power management because the web applications are becoming more sophisticated and their processing power is gradually increasing. Current cluster are designed to handle peak loads, where all servers are equally utilized. In practice, peak load conditions barely happen and clusters are most of the time underutilized. This paper aims to optimize the performance (in terms of execution time) of a multi-tier system and decrease the power consumption of the servers. To achieve these objectives, an Energy Optimizing Genetic Algorithm (EOGA) technique is applied on a three-tier web cluster system. This technique has proved to be successful in decreasing the power consumption and increasing the performance of the system.
\end{abstract}

\section{Keywords}

Genetic algorithm, Heterogeneous, Multi-tier cluster, Power, Server.

\section{INTRODUCTION}

The web applications which are complex usually get their service from the multi-tier web clusters. For example, a three tier web cluster is comprised of web or presentation tier, application tier and a database tier. Every tier performs differently in different applications. Power management is the process of reducing consumption of power taking into account various parameters. Many techniques have been proposed for power management for large scale distributed systems [29, 30, 31]. As the cost of power is growing day-by-day, more attention is being paid towards the power management by the researchers $[1,2,3-5,8]$. To meet the target response delay constraints even under the peak loads, these web servers are often over-provisioned significantly which leads to incur low energy efficiency for those clusters. Therefore, it is desired to increase performance per unit of energy. Several power management techniques have been proposed for multi-tier clusters [9-14]. Most of the researches have focused on homogeneous clusters [22-24]. Clusters are invariably different in terms of their capacity, power consumption and performance. Nowadays, data centers are mostly comprised of heterogeneous servers so that the failed servers could be replaced with others easily. In the heterogeneous systems when any upgrades are made to the data center, the replacement of all the servers is technically very much difficult because of the size of the data center. Due to this reason, new servers having different specifications are attached to the previous ones. New challenges are introduced by the heterogeneous architecture to the power management of clusters. In case of the homogeneous clusters, we were only required to be familiar with the number of servers to be turned on and with which frequency level they should run at. On the other hand, a decision is to be made on which server should be powered on or off in case of heterogeneous clusters. Apart from that, the distribution of workload is not an easy task in the heterogeneous clusters which was not a big of an adversity when it comes to homogeneous clusters.

\section{RELATED WORK}

In order to decide the best and fruitful power management technique for this multi-tier web cluster model, the below given literature review was surveyed.

Sharma et al. [19] investigated some algorithms for dynamic voltage scaling in QoS-enabled web servers to reduce the consumption of energy that subject to service delay constraints. Algorithms were implemented inside the Linux kernel. The consumption of energy was reduced by using a feedback loop.

N. Wang [25] carried out the performance of parallel optimization with the help of genetic algorithm (GA) for lubrication analysis. The author used an air-bearing design as an example and he carried out the parallel computation in a single system image (SSI) cluster.

Kolodjeij et. al [26] presented the independent batch scheduling approach as a bi-objective minimization problem with makespan and energy consumption as the scheduling criteria. Dynamic Voltage Scaling (DVS) methodology for reduction of power energy by the resources and scaling was used. Authors developed two implementations of Hierarchical Genetic Strategy-based grid scheduler (Green-HGS-Sched) with struggle replacement methods.

N. Kaur et al. [27, 28] discussed several power management and power reduction techniques for reducing the overall energy consumption of the system. Authors also discussed the energy aware scheduling techniques based on list duplication and clustering based heuristics approaches to reduce the energy consumption and overall makespan.

\subsection{Power management for multi-tier clusters}

Horvath et al. [5] presented a DVS control algorithm that minimizes power consumption in a server pipeline subject to end-to-end latency constraints. Their implementation had two components: an Apache module to measure the end-to-end latencies and a daemon on each server that measured its CPU utilization ran the feedback controller.

Horvath et al. [6] stated a policy for the energy management for the clusters running a multi tier applications thereby exploiting the DVS with various sleep states. A theoretical 
analysis of the power optimization problem and an algorithm for the solution were designed and developed. Also the selection of optimal number of spare servers per power state was investigated. In order to validate the results and policies, all the power management techniques supporting clusters were considered.

Choi et al. [1] identified power budgets of two kinds (i) a sustained budget to capture any restrictions above a certain threshold. (ii) An average budget to capture an upper bound on long term energy being consumed. The power profiles statistical description of the power being consumed by the applications was derived with the help of a simple measurement infrastructure. Also the models for predicting the sustained and average power being consumed by the applications were developed.

Govindan et al. [15] explored a combination of statistical multiplexing techniques to improve the utilization of the power hierarchy within a data center. At the highest level of the power hierarchy, they employed a controlled under provisioning and over-booking of power needs of hosted workloads. At the lower levels, they introduced the novel notion of soft fuses to flexibly distribute provisioned power among hosted workloads based on their needs.

Rao et al. [8] studied that while guaranteeing the quality of service, minimizing the total electricity was a severe problem. Therefore, an efficient solution method was proposed considering the problem as mixed integer programming and the evaluations based on a real life electricity price data for multiple locations was conducted.

Schranzhofer et al. [13] stated that the key steps in the design of efficient embedded systems were task assignment and processing unit allocation. A methodology for the computation of task to process element mapping was proposed in order to minimize the expected average power being consumed.

\subsection{Power management for homogeneous clusters}

Zong et al. [24] presented two energy efficient scheduling algorithms based on duplication that are Energy-Aware Duplication (EAD) scheduling and Performance-Energy Balanced Duplication (PEBD) scheduling to optimize performance as well as energy efficiency. Authors investigated that power can be saved through power aware duplication based scheduling for both interconnect and processors. DVFS was used to save energy consumption in processors.

Liu et al. [22] proposed an Energy-efficient Clustering based Scheduling algorithm for parallel tasks on homogeneous DVS-enabled clusters (ECSTD) that helps to improve both energy efficiency and performance in homogeneous clusters. Algorithm was also based on threshold which was controllable and can be varied to get the optimal balance between the energy saving effect and performance. The delay of network was considered during the scheduling because the actual network environment differs from the simulation network environment.

Zhao et al. [23] proposed an energy-efficient Dependencybased task Grouping (DG) method to assign parallel tasks under precedence constrains to multi-core processors which helps in optimizing the energy efficiency and performance in homogeneous clusters.

\subsection{Power management for heterogeneous clusters}

Rusu et al. [12] stated a cluster wide QoS-aware technique to decrease energy consumption during periods of reduced loads. The effects of local QoS-aware power management using DVS were also investigated. The implementation of the algorithm was described and evaluated using Apache web server for validation in small realistic cluster.

Al-Daoud et al. [9] proposed LPAS policy for heterogeneous clusters. The experimentation results showed the reduction in energy consumption. A scheduling policy was applied to their workload model. For major schedulers, FCFS was used. Pickthe-Most-Efficient (PME) policy was used as another candidate policy. This policy worked as greedy approach for assigning tasks to machines. Then LPAS policy was used for solving two linear programming (LP) problems.

Wang et al. [16] addressed the problem of managing power in heterogeneous multi tier web clusters. Generalized Benders Decomposition (GBD) technique was applied to divide the global optimization problem into sub problems. This technique worked in an iterative fashion and helped in achieving more energy consumption.

Remaining paper is organized as Section 3 describes the system model. Section 4 describes the proposed technique. Section 5 represents the experimental results and simulation. Section 6 represents the acknowledgement. Section 7 shows the conclusion and future scope of the work implemented. Section 8 shows references.

\section{SYSTEM MODEL}

\subsection{Web cluster model}

I studied a general three-tier web cluster model in which the first tier depicts web service to the client, the second tier depicts the business logic and the third tier depicts the database tier as shown in figure 1 .

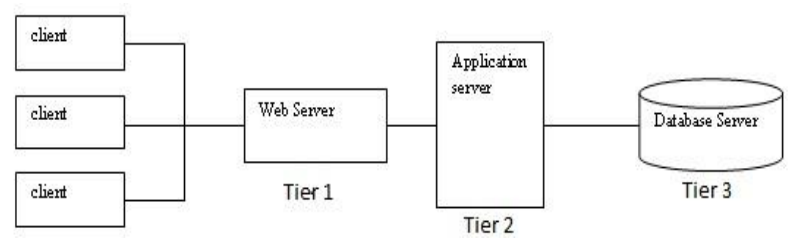

Fig 1: A typical three-tier web cluster.

\subsection{Power model}

In this paper, power is optimized using energy optimizing genetic algorithm technique. Mathematically,

For $\mathrm{i}=1$ to 2

Fitness $($ loop_val $=i)=S_{i}($ fitness_fn $(F s, F t))$

Where

$\mathrm{S}_{\mathrm{i}}=$ for a particular value.

Fs $=$ current_job_power.

$\mathrm{Ft}=$ avg_power_per_server.

\section{ENERGY OPTIMIZING GENETIC ALGORITHM}

Energy Optimizing Genetic Algorithm (EOGA) is a method to optimize the power consumption and performance (in terms of execution time) in heterogeneous multi-tier web cluster 
systems. In EOGA, power consumed by the job is the parameter which has to be optimized. Population taken is 50, mutation operator value is 0.05 and crossover operator value is 0.8 .

\subsection{Flowchart of EOGA}

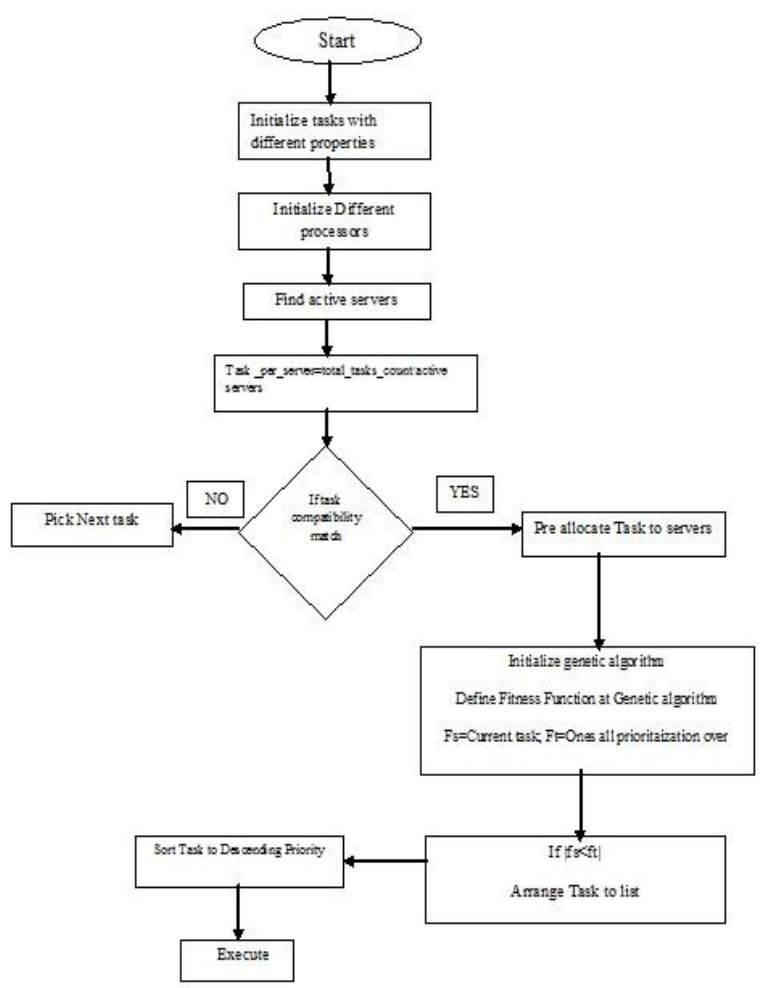

Fig 2: Flowchart of Energy Optimizing Genetic Algorithm technique.

First, initialize the task with different properties like load, power, id and its state and then initialize the server with its alive state, its power and load and the time for server to simulate it in milliseconds. Find the active servers and the task per server with total count active server. If the task compatibility match then allocates the tasks to the active servers. Then optimize the power of the server using energy optimizing genetic algorithm technique. Initialize the EOGA technique using population size and its operators in which the fitness function is designed where the power of the current task is compared with the total power. If the current task computes less power than total power then update the list of the tasks, sort task to the descending priority and execution process will takes place.

\subsection{EOGA pseudo code}

1. START

2. INITIALIZE servers with its load, power, state and id.

3. INPUT number of Servers, execution time.

4. Do

5. Check power $=\mathrm{p}$

6. Check load $=1$

7. Check jobs $=\mathrm{j}$

8. FOR $\mathrm{i}=1$ to number of iterations for all the input process applied to the main program.

9. Check the number of servers in alive state.

10. Generate job, $\mathrm{j}$ specifications.

11. Allocate the jobs to the alive servers.

12. REPEAT step 5, 6 and 7.

13. INITIALIZE energy optimizing genetic algorithm.

14. INITIALIZE the function with population size 50 , mutation value 0.05 and crossover value 0.8 .

15. REPEAT for the number of jobs entered.

16. Call fitness function with the parameters Fs = current_job_power and $\mathrm{Ft}=$ average_power_per_server.

17. IF Ft is less than the Fs.

18. THEN reduce Fs.

19. ENDIF

20. REPEAT UNTIL the number of jobs entered completed.

21. Check the number of jobs executed.

22. Check the average power consumed.

23. ENDFOR

24. STOP

\section{RESULTS AND SIMULATION}

This section presents the results of the proposed technique. MATLAB is used for implementation of the algorithm and simulation of clusters. The program runs on Intel(R) core i3 CPU $2.27 \mathrm{GHz}$ and 3G RAM. Servers are taken randomly considering following parameters:-

Table 1. Server Parameters

\begin{tabular}{|c|c|c|}
\hline S. No. & Parameters & Values \\
\hline 1. & Server id & s_id \\
\hline 2. & Server load & s_load \\
\hline 3. & Server state & s_state \\
\hline 4. & Server power & s_power \\
\hline
\end{tabular}

Two scenarios are considered in this paper. In first scenario, 15 servers were taken for the simulation time of 10 milliseconds. There were 9 active servers out of 15 servers in this scenario. Table 2 represents the percentage of job completion with respect to number of job requests in the first scenario.

Table 2. Percentage of job completion in scenario $I$.

\begin{tabular}{|c|c|c|}
\hline S. No. & Job Requests & EOGA \\
\hline 1. & 1000 & $2.7 \%$ \\
\hline 2. & 2000 & $1.45 \%$ \\
\hline
\end{tabular}




\begin{tabular}{|l|l|l|}
\hline 3. & 3000 & $0.86 \%$ \\
\hline 4. & 4000 & $0.65 \%$ \\
\hline 5. & 5000 & $0.46 \%$ \\
\hline
\end{tabular}

PERCENTAGE OF THE COMPLETION OF JOBS 0.4GAT ITERATION NUMBER:5 WITH JOE FLOW:5000

\section{OK}

Fig 3: Percentage of job completion in scenario $I$.

Figure 3 represents the percentage completion of jobs with respect to number of iterations and results that at $5^{\text {th }}$ iteration the completion job percentage is $0.46 \%$ with 5000 job requests.

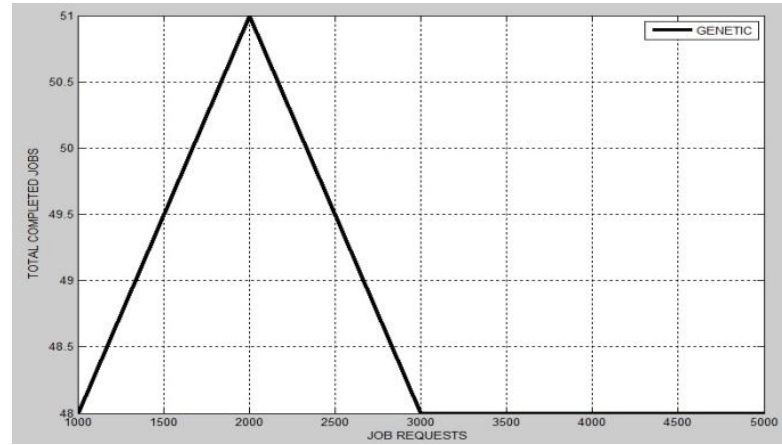

Fig 4: Total number of jobs completed in scenario I.

Figure 4 represents the total number of jobs completed with respect to the number of requested jobs using EOGA in scenario I.

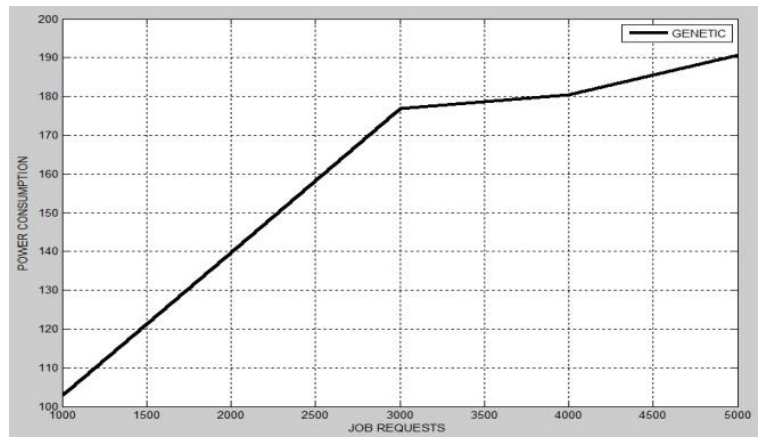

Fig 5: Average power consumption in scenario I.

Figure 5 represents the average energy consumption using EOGA which is $192 \mathrm{~W}$ with respect to the number of requested jobs.

Table 3. Average power consumption in scenario I.

\begin{tabular}{|c|c|c|}
\hline S. No. & Jobs requests & EOGA \\
\hline 1. & 5000 & $192 \mathrm{~W}$ \\
\hline
\end{tabular}

In second scenario, 18 servers were taken for the simulation time of 12 milliseconds. There were 12 active servers out of 18 servers in this scenario. Table 4 represents the percentage of job completion with respect to number of job requests in second scenario.
Table 4. Percentage of job completion in scenario II.

\begin{tabular}{|c|c|c|}
\hline S. No. & Job Requests & EOGA \\
\hline 1. & 1000 & $3.5 \%$ \\
\hline 2. & 2000 & $1.9 \%$ \\
\hline 3. & 3000 & $1.03 \%$ \\
\hline 4. & 4000 & $1.05 \%$ \\
\hline 5. & 5000 & $0.7 \%$ \\
\hline
\end{tabular}

PERCENTAGE OF THE COMPLETION OF JOBS 0.7AT ITERATION NUMBER:5 WITH JOB FLOW:5000

\section{OK}

Fig 6: Percentage of job completion in scenario II.

Figure 6 represents the percentage completion of jobs with respect to number of iterations and results that at $5^{\text {th }}$ iteration the completion job percentage is $0.70 \%$ with 5000 job requests.

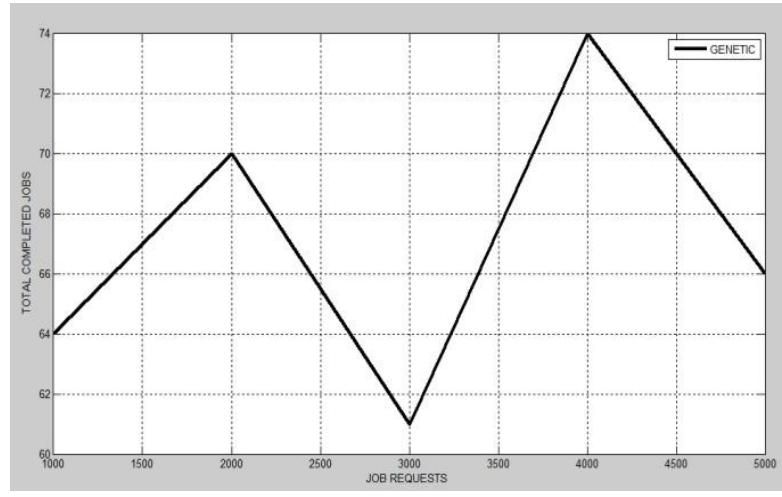

Fig 7: Total number of jobs completed in scenario II.

Figure 7 represents the total number of jobs completed with respect to the number of requested jobs using EOGA in scenario II.

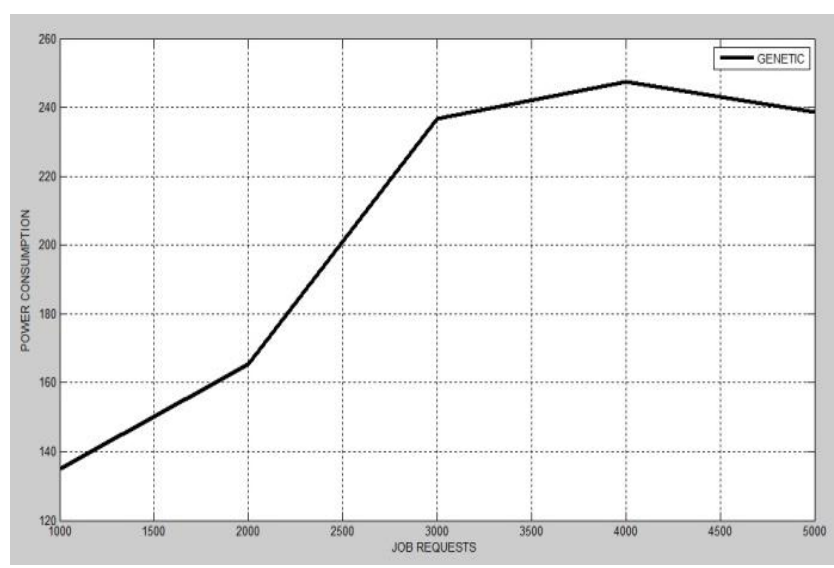

Fig 8: Average power consumption in scenario II.

Figure 8 represents the average energy consumption using EOGA which is $248 \mathrm{~W}$ with respect to the number of requested jobs. 
Table 5. Average power consumption in scenario II.

\begin{tabular}{|l|l|l|}
\hline S. No. & Jobs requests & EOGA \\
\hline 1. & 5000 & $248 \mathrm{~W}$ \\
\hline
\end{tabular}

\section{CONCLUSION AND FUTURE WORK}

This paper aims to increase the performance (in terms of execution time) and decrease the power consumption in heterogeneous multi-tier web clusters. MATLAB simulation tool was used in order to perform the implementation. This paper consists of two scenarios. In first scenario, 15 servers were taken for the simulation time of 10 milliseconds. The power consumption by the servers was 192 Watts and job completion percentage was $46 \%$ having 5000 job requests. In second scenario, 18 servers were taken for the simulation time of 12 milliseconds. In this scenario, the power consumption by the servers was 248 Watts and job completion percentage was $70 \%$ having 5000 job requests.

Further research can be performed in order to increase the performance and reduce the power consumption. Better problem optimizing techniques can be applied. Better energy efficiency or scheduling techniques can be inculcated.

\section{ACKNOWLEDGMENT}

I would like to express my sincere gratitude towards my thesis guide Ms. Nirmal Kaur who helped and guided me to complete this paper successfully.

\section{REFERENCES}

[1] J. Choi, S. Govindan, B. Urgaonkar, A. Sivasubramaniam, "Profiling, prediction, and capping of power consumption in consolidated environments," in: International Symposium on Modeling, Analysis and Simulation of Computers and Telecommunication Systems, MASCOTS, IEEE, pp. 1-10, 2008.

[2] X. Fan, W. Weber, L. Barroso, "Power provisioning for a warehouse-sized computer," in: Proceedings of the 34th Annual International Symposium on Computer Architecture, Association for Computing Machinery, Inc., New York, NY, pp. 13-23, 2007.

[3] J. Heo, D. Henriksson, X. Liu, T. Abdelzaher, "Integrating adaptive components: an emerging challenge in performance-adaptive systems and a server farm case-study," in: 28th IEEE International Real-Time Systems Symposium, RTSS, IEEE, pp. 227-238, 2007.

[4] J. Heo, P. Jayachandran, I. Shin, D. Wang, T. Abdelzaher, X. Liu, "Optituner: on performance composition and server farm energy minimization application,” IEEE Trans. Parallel Distrib. Syst. 22 (11), pp. 1871-1878, 2011.

[5] T. Horvath, T. Abdelzaher, K. Skadron, X. Liu, "Dynamic voltage scaling in multitier web servers with end-to-end delay control," IEEE Trans. Comput. 56 (4), pp. 444-458, 2007.

[6] T. Horvath, K. Skadron, "Multi-mode energy management for multi-tier server clusters," in: Proceedings of the 17th International Conference on Parallel Architectures and Compilation Techniques, ACM, pp. 270-279, 2008.

[7] T. Horvath, K. Skadron, T. Abdelzaher, "Enhancing energy efficiency in multitier web server clusters via prioritization," in: IEEE International Parallel and Distributed Processing Symposium, IEEE, pp. 1-6, 2007.

[8] L. Rao, X. Liu, L. Xie, W. Liu, "Minimizing electricity cost: optimization of distributed Internet data centers in a multi-electricity-market environment," in: Proceedings IEEE INFOCOM, IEEE, pp. 1-9, 2010.

[9] H. Al-Daoud, I. Al-Azzoni, D. Down, "Power-aware linear programming based scheduling for heterogeneous computer clusters," Future Generation Computer Systems 28, pp. 745-754, 2012.

[10] T. Heath, B. Diniz, E. Carrera, W. Meira Jr., R. Bianchini, "Energy conservation in heterogeneous server clusters," in: Proceedings of the tenth ACM SIGPLAN Symposium on Principles and Practice of Parallel Programming, ACM, pp. 186-195, 2005.

[11] Krioukov, P. Mohan, S. Alspaugh, L. Keys, D. Culler, R. Katz, "Napsac: design and implementation of a powerproportional web cluster," ACM SIGCOMM Comput. Commun. Rev. 41 (1), pp. 102-108, 2011.

[12] Rusu, A. Ferreira, C. Scordino, A. Watson, "Energyefficient real-time heterogeneous server clusters," in: Real-Time and Embedded Technology and Applications Symposium, IEEE, pp. 418-428, 2006.

[13] Schranzhofer, J. Chen, L. Thiele, "Dynamic poweraware mapping of applications onto heterogeneous mpsoc platforms," IEEE Trans. Ind. Inform. 6 (4), pp. 692-707, 2010.

[14] L. Wang, Y. Lu, "An efficient threshold-based power management mechanism for heterogeneous soft real-time clusters," IEEE Trans. Ind. Inform. 6 (3), pp. 352-364, 2010 .

[15] S. Govindan, J. Choi, B. Urgaonkar, A. Sivasubramaniam, A. Baldini, "Statistical profilingbased techniques for effective power provisioning in data centers," in: Proceedings of the 4th ACM European Conference on Computer systems, ACM, pp. 317-330, 2009.

[16] P. Wang, Y. Qi, X. Liu, Y. Chen, X. Zhong, "Power management in heterogeneous multi-tier web clusters," in: J. Parallel Distrib. Comput.74, pp. 2005-2015, 2014.

[17] E.N. Elnozahy, Michael Kistler, Ramakrishnan Rajamony, "Energy-efficient server clusters," in: Proceedings of the Second International Workshop of Power-Aware Computer Systems, pp. 179-196, 2002.

[18] Eduardo Pinheiro, Ricardo Bianchini, Enrique V. Carrera, Taliver Heath, "Dynamic cluster reconfiguration for power and performance," in: Compilers and Operating Systems for Low Power, Kluwer Academic Publishers, pp. 75-93, 2003.

[19] Vivek Sharma, Arun Thomas, Tarek Abdelzaher, Kevin Skadron, Zhijian Lu, "Power-aware QoS management in web servers," in: Proceedings of the $24^{\text {th }}$ International Real-Time Systems Symposium, pp. 63-72, 2003.

[20] Mootaz Elnozahy, Michael Kistler, Ramakrishnan Rajamony, "Energy conservation policies for web servers," in: Proceedings of the 4th conference on USENIX Symposium on Internet Technologies and Systems, USENIX Association, 2003.

[21] Fabien Hermenier, Xavier Lorca, Jean-Marc Menaud, 
Gilles Muller, Julia Lawall, "Entropy: a consolidation manager for clusters," in: Proceedings of the ACM SIGPLAN/SIGOPS International Conference on Virtual Execution Environments (VEE '09), pp. 41-50, 2009.

[22] Wei Liu, Yuguang Duan, Wei Du, “An Energy Efficient Clustering-based Scheduling Algorithm for Parallel Tasks on Homogeneous DVS-Enabled Clusters," in: Proceedings of the IEEE 16th International Conference on Computer Supported Cooperative Work in Design, pp. $575-582,2012$

[23] Yanheng Zhao, Xin Li, Zhiping Jia, Lei Ju, Ziliang Zong, "Dependency-based Energy-Efficient Scheduling for Homogeneous Multi-core Clusters," in: Proceedings of 12th IEEE International Conference on Trust, Security and Privacy in Computing and Communications, pp. 1299-1306, 2013.

[24] Ziliang Zong, Adam Manzanares, Xiaojun Ruan, and Xiao Qin, "EAD and PEBD: Two Energy-Aware Duplication Scheduling Algorithms for Parallel Tasks on Homogeneous Clusters," IEEE transaction on computers, vol. 60(3), pp. 360-374, 2011

[25] Wang, Nenzi. "A parallel computing application of the genetic algorithm for lubrication optimization." Tribology Letters 18, no. 1, pp. 105-112, 2005.

[26] Kołodziej, Joanna, Samee Ullah Khan, Lizhe Wang, Aleksander Byrski, Nasro Min-Allah, and Sajjad Ahmad
Madani. "Hierarchical genetic-based grid scheduling with energy optimization." Cluster Computing 16, no. 3, pp. 591-609, 2013.

[27] Nirmal Kaur, Savina Bansal, R. K. Bansal, ”Energy aware scheduling strategies for distributed computing systems," International journal of advanced research in computer science and software engineering, vol. 3, issue 10, pp. 280-283, 2013

[28] Nirmal Kaur, Savina Bansal, R. K. Bansal, "Task scheduling and energy conservation techniques for multiprocessor computing systems," International journal of networks and systems, vol. 2, no. 2, pp. 5-8, 2013.

[29] Anne Cecile Orgerie, Marcos Dias De Assuncao, Laurent Lefevre, "A survey on techniques for improving energy efficiency of large scale distributed systems," ACM Computing Surveys, vol. TBD, no. TBD, pp. 1-35, 2013.

[30] Luca Benini, Alessandro Bogliolo, Giovanni De Micheli, "A survey of design techniques for system-level dynamic power management," IEEE transactions on very large scale integration (VLSI) systems, vol 8, no. 3, pp. 289316, 2000.

[31] Sergey Zhuravlev, Juan Carlos Saez, Sergey Blagodurov, Alexandra Fedorova and Manuel Prieto, "Survey of energy-cognizant scheduling techniques," IEEE transactions on parallel and distributed systems, vol. 24, no. 7, pp. 1447-1463, 2013. 